Volume 141, Number 12, December 2013, Pages 4071-4085

S 0002-9939(2013)12025-3

Article electronically published on August 30, 2013

\title{
SPIDERS AND MULTIPLICITY SEQUENCES
}

\author{
SHREERAM S. ABHYANKAR AND IGNACIO LUENGO
}

(Communicated by Lev Borisov)

\begin{abstract}
The spider principle is used for establishing a formula for a finite quadratic sequence which determines the multiplicity sequences of all the sprouts which are founded upon the given finite quadratic sequence. This formula is basic for the theories of curvettes and dicriticals.
\end{abstract}

\section{INTRODUCTION}

The "spider principle" says that if a point in the support of a two variable power series does not lie on its Newton polygon, then it cannot get in after a QDT $=$ Quadratic Transformation. This principle originated in our previous paper AL1, where it was used for giving an algebraic proof of the fact that the dicritical divisors of a special pencil have a certain polynomial property. In the analytic situation, this was proved by Artal Bartolo Art by topological methods. In this paper we shall use the spider principle for establishing a multiplicity formula for finite QDT sequences. This formula will play a significant role in a forthcoming paper of Abhyankar-Artal AA1, where curvettes will be employed in answering a question about the existence of dicriticals in the case of two dimensional regular local domains raised in Ab12. In the Abhyankar-Heinzer paper AH2, which is a sequel to their paper [AH1, the question is dealt with in case of higher dimensions by employing the algebra of Rees rings.

Briefly speaking, referring to the next section for notation, the said formula can be described thus. Let $S=\left(S_{j}, x_{j}, y_{j}, \kappa_{j}\right)_{0 \leq j \leq \nu}$ be any finite QDT sequence of $(R, x, y, \kappa)$ where $x, y$ are generators of the maximal ideal $M(R)$ of a two dimensional regular local domain $R$ and $\kappa$ is a coefficient set of $R$. The characteristic of the residue field of $R$ is allowed to be different from the characteristic of $R$. To $S$ associate the "word" $W=W_{1} W_{2} \ldots W_{\nu}$ in the free group on three generators $X, Y, Z$ given by $W_{j}=X$ or $Y$ or $Z^{\chi\left(S_{j-1}, S_{j}\right)}$ according as $j$ is an $X$-operation or $Y$-operation or a translation of $S$, where $\chi\left(S_{j-1}, S_{j}\right)$ denotes the field degree of $S_{j} / M\left(S_{j}\right)$ over $S_{j-1} / M\left(S_{j-1}\right)$. Assume that $\nu$ is a translation of $S$ or, more generally, assume that the Newton polygon of a generator of $I_{\nu-1}$ is a triangle of a certain slope. Then $W$ determines a multiplicity sequence of positive integers $m_{0} \geq m_{1} \geq \cdots \geq m_{\nu-1}=1$ having the following property: If $I_{j}$ is any nonzero nonunit unitangent principal ideal in $R_{j}$ such that $I_{j+1}$ is the (proper) transform

Received by the editors February 1, 2012.

2010 Mathematics Subject Classification. Primary 14A05.

Key words and phrases. Dicritical, multiplicity sequence, spider principle.

The second author was partially supported by MTM2010-21740-C02-01. 
of $I_{j}$ in $R_{j+1}$, then we have

$$
\operatorname{ord}_{R_{j}} I_{j}=m_{j} \operatorname{ord}_{R_{\nu-1}} I_{\nu-1} \text { for } 0 \leq j \leq \nu-1 .
$$

In Sections 2 and 3 we deal with the case when $\nu$ is a translation of $S$. In Sections 4 and 5 we deal with the more general case where the multiplicity sequence extends through $\nu$ and may not end with the value 1; i.e., it will simply be a sequence of positive integers $m_{0} \geq m_{1} \geq \cdots \geq m_{\nu}$. These sequences depend only on the word $W$ and not on any ideals $I_{j}$ or even on the the local ring $R$ or its characteristic.

\section{Notation}

In general we shall follow the terminology of Ab8] to $\mathrm{Ab14}$, especially that of Section 3 of [Ab9], Sections 2 to 4 of [Ab10], Section 2 and Remark (4.0) of Ab11, Sections 2 and 3 of Ab12, Sections 2 to 4 of Ab13, and Sections 2 to 5 of Ab14. Some other basic references are $\mathrm{Ab1}$ to $\mathrm{Ab7}$.

Recall that $H_{B}: B \rightarrow H(B)=B / M(B)$ denotes the residue class epimorphism of any quasilocal ring $B$, and for any subring $A$ of $B$ let us put

$$
\chi(A, B)=\left[K^{\prime}: K\right],
$$

where $K^{\prime}$ is the algebraic closure of $K=\mathrm{QF}\left(H_{B}(A)\right)$ in $H(B)$.

Let $R$ be a two dimensional regular local domain with quotient field $\Omega$, residue field $K=H(R)$, generators $(x, y)$ of $M(R)$, and coefficient set $\kappa$ of $R$.

Let $S=\left(S_{j}, x_{j}, y_{j}, \kappa_{j}\right)_{0 \leq j \leq \nu}$ be any finite QDT sequence of $(R, x, y, \kappa)$. In the third paragraph of Section 5 of [Ab14], after introducing the terms height $h(S)=\nu$, $X$-operation, $Y$-operation, translation, nontranslation, translation index $t(S) \in \mathbb{N}$, preflip, $X$-preflip, $Y$-preflip, $X$-flip, $Y$-flip, protoflip, retroflip, pillar, nonflip, flip, pillar number $n(S) \in \mathbb{N}$, top, bottom, head, and tail, the concept of the flip sequence of $S$ was introduced by saying:

"We define the pillar length of $S$ to be a positive integer $l(S)$, its height sequence $q(S)=q_{i}(S)_{0 \leq i \leq l(S)}$ where $q_{i}(S) \in \mathbb{N}$ with $0=q_{0}(S) \leq \cdots \leq q_{l(S)}(S)$ and $q_{l(S)-1}(S)=t(S) \leq \nu=q_{l(S)}(S)$ with $l(S)>2 \Rightarrow q_{l(S)-2}(S)<q_{l(S)-1}(S)$, and its flip sequence $\left(S^{(i)}\right)_{1 \leq i \leq l(S)}$ where

$$
S^{(i)}=\left(S_{q_{i-1}(S)+j}, x_{q_{i-1}(S)+j}, y_{q_{i-1}(S)+j}, \kappa_{q_{i-1}(S)+j}\right)_{0 \leq j \leq q_{i}(S)-q_{i-1}(S)}
$$

for $1 \leq i<l(S)$ is a nonflip or flip at $\left(S_{q_{i-1}(S)}, x_{q_{i-1}(S)}, y_{q_{i-1}(S)}, \kappa_{q_{i-1}(S)}\right)$ according as $i$ is odd or even and $S^{(l(S))}=$ the head of $S . "$

(2A) Our Goal. In the case of $t(S)>0$, we shall introduce one more invariant of $S$, namely its multiplicity sequence $m(S)=m_{j}(S)_{1 \leq j \leq t(S)-1}$ with integers $m_{0}(S) \geq \cdots \geq m_{t(S)-1}(S)=1$. First in (2B), as a preparation for flips and nonflips, we shall introduce auxiliary multiplicity sequences $m_{j}^{\prime}$ together with auxiliary glue bisequences $\left(a_{j}^{\prime}, b_{j}^{\prime}\right)$. Then, for the general case of $t(S)>0$, the sequences $m_{j}, a_{j}, b_{j}$ themselves will be defined in (2C) and redone in Section 5. Important properties of these sequences will be stated in (2D) and proved in Sections 3 and 4.

(2B) Preparation. Let $N_{\nu}$ be the set of all positive integers $\leq \nu$, and let $\bar{N}_{\nu}=$ $\{0\} \cup\left(N_{\nu} \backslash\{\nu\}\right)$. Let $\{X, Y, Z\}$ be indeterminates, i.e., a set of size three. By the label of $S$ we mean the map $\lambda(S): N_{\nu} \rightarrow\{X, Y, Z\}$ given by $j \mapsto \lambda_{j}(S)=X$ or $Y$ or $Z$ according as $j$ is an $X$-operation or $Y$-operation or a translation of $S$. 
For any map $\lambda: N_{\nu} \rightarrow\{X, Y, Z\}$ such that $\nu \geq 2$ with $\lambda_{j}=Y$ for some positive integer $j<\nu$ and $\lambda_{j} \neq Z$ for $1 \leq j<\nu$ but $\lambda_{\nu}=Z$, we define the derivative as the map $\lambda^{\prime}: \bar{N}_{\nu} \rightarrow \mathbb{N}_{+}$given by $j \mapsto \lambda_{j}^{\prime}$, with integers $\lambda_{0}^{\prime} \geq \cdots \geq \lambda_{\nu-1}^{\prime}=1$, in the following manner. Referring to the first ten lines of Section 3 of Ab9, but restricting our attention to nonnegative integers, we define a euclidean sequence as a sequence $\left(e_{i}, p_{i}\right)_{0 \leq i \leq \epsilon}$, where $e_{j} \in \mathbb{N}_{+}$and $p_{j} \in \mathbb{N}$ with $3 \leq \epsilon \in \mathbb{N}_{+}$are such that:

(1) $p_{0}=0 \neq p_{j}$ for $2 \leq j \leq \epsilon$,

(2) $e_{j-1}=p_{j} e_{j}+e_{j+1}$ with $0<e_{j+1}<e_{j}$ for $1 \leq j \leq \epsilon-1$,

(3) $e_{j}>e_{\epsilon}=1=\operatorname{GCD}\left(e_{0}, e_{1}\right)=\operatorname{GCD}\left(e_{0}, \ldots, e_{\epsilon}\right)$ for $1 \leq j \leq \epsilon-1$,

(4) $e_{0} \not \equiv 0 \bmod \left(e_{1}\right)$ and $e_{\epsilon-1}=p_{\epsilon} e_{\epsilon}$.

Clearly, for any $\lambda$ as above, there exists a unique euclidean sequence $\left(e_{i}, p_{i}\right)_{0 \leq i \leq \epsilon}$ such that:

$\lambda_{j}=X$ for $p_{0}+\cdots+p_{u-1}<j \leq p_{0}+\cdots+p_{u}$ for odd positive integers $u \leq \epsilon$ and

$\lambda_{j}=Y$ for $p_{0}+\cdots+p_{u-1}<j \leq p_{0}+\cdots+p_{u}$ for even positive integers $u \leq \epsilon$ and

We put

$$
p_{0}+\cdots+p_{\epsilon}=\nu-1 \text { with } \lambda_{\nu}=Z \text {. }
$$

and

$$
\lambda_{j}^{\prime}=e_{j} \text { for } p_{0}+\cdots+p_{u-1}<j \leq p_{0}+\cdots+p_{u} \text { for positive integers } u \leq \epsilon .
$$

Assuming $S$ to be a flip, taking $\lambda(S)$ for $\lambda$, we put: $\lambda^{\prime}(S)=\lambda^{\prime}$ with $\lambda_{j}^{\prime}(S)=\lambda_{j}^{\prime}$ for $0 \leq j \leq \nu-1, \epsilon(S)=\epsilon, e_{i}(S)=e_{i}$ and $p_{i}(S)=p_{i}$ for $0 \leq i \leq \epsilon$, and now as a preparation for introducing the multiplicity sequence of $S$, we define positive integers $m_{j}^{\prime}(S)_{0 \leq j \leq \nu-1}$ with $m_{0}^{\prime}(S) \geq \cdots \geq m_{\nu-1}^{\prime}(S)=1$ by letting $m_{j}^{\prime}(S)=\lambda_{j}^{\prime}(S)$ for $0 \leq j \leq \nu-1$.

Likewise, assuming $S$ to be a nonflip with $\nu>0$, as a preparation for introducing the multiplicity sequence of $S$, we define positive integers $m_{j}^{\prime}(S)_{0 \leq j \leq \nu-1}$ with $m_{0}^{\prime}(S) \geq \cdots \geq m_{\nu-1}^{\prime}(S)=1$ by letting $m_{j}^{\prime}(S)=\chi\left(S_{j}, S_{\nu-1}\right)$ for $0 \leq j \leq \nu-1$.

As an aid to defining the multiplicity sequence for the general case of $t(S)>0$, we define a bisequence $\left(a_{i}(S), b_{i}(S)\right)_{0 \leq i \leq t(S)-1}$ of positive integers which we call the glue bisequence of $S$.

Assuming $S$ to be either a flip or a nonflip with $\nu>0$, to prepare for introducing the glue bisequence of $S$, let us define positive integers $\left(a_{j}^{\prime}(S), b_{j}^{\prime}(S)\right)_{0 \leq j \leq \nu-1}$ in the following manner. (i) If there exists an integer $\nu^{\prime} \geq 2$ with $j+\nu^{\prime} \leq \nu$ such that $S^{\prime}=\left(S_{j+k}, x_{j+k}, y_{j+k}, \kappa_{j+k}\right)_{0 \leq k \leq \nu^{\prime}}$ is a flip, then we put $\left(a_{j}^{\prime}(S), b_{j}^{\prime}(S)\right)=$ $\left(e_{1}\left(S^{\prime}\right), e_{0}\left(S^{\prime}\right)\right)$, and note that $\nu^{\prime}$ is then unique. (ii) On the other hand, if there exists an integer $\nu^{\prime} \geq 1$ with $j+\nu^{\prime} \leq \nu$ such that $S^{\prime}=\left(S_{j+k}, x_{j+k}, y_{j+k}, \kappa_{j+k}\right)_{0 \leq k \leq \nu^{\prime}}$ is a nonflip, then, upon letting $\nu^{\prime \prime}$ be the smallest positive integer $\leq \nu^{\prime}$ for which $\nu^{\prime \prime}$ is a translation of $S^{\prime}$, we put $\left(a_{j}^{\prime}(S), b_{j}^{\prime}(S)\right)=\left(m_{0}^{\prime}\left(S^{\prime}\right), m_{0}^{\prime}\left(S^{\prime}\right) \nu^{\prime \prime}\right)$, and note that $\nu^{\prime}$ and $\nu^{\prime \prime}$ are then unique.

(2C) Definition. Modify the above quote "We define ... head of $S$ " just before (2A) by writing $q_{i}$ for $q_{i}(S)$. In the general case of $t(S)>0$, using the equality $q_{l(S)-1}=$ $t(S)$ together with the implication $l(S)>2 \Rightarrow q_{l(S)-2}<q_{l(S)-1}$ from the modified 
quote, the multiplicity sequence $m(S)=m_{j}(S)_{1 \leq j \leq t(S)-1}$ of $S$, together with its glue bisequence $\left(a_{j}(S), b_{j}(S)\right)_{0 \leq j \leq t(S)-1}$, is defined by the recurrence formulas

$$
\left\{\begin{array}{c}
m_{j-q_{i-1}}(S)=m_{j-q_{i-1}}\left(S^{(i)}\right) a_{q_{i}}(S) \chi\left(S_{q_{i}-1}, S_{q_{i}}\right), \\
a_{j-q_{i-1}}(S)=a_{j-q_{i-1}}\left(S^{(i)}\right) a_{q_{i}}(S) \chi\left(S_{q_{i}-1}, S_{q_{i}}\right), \\
b_{j-q_{i-1}}(S)=b_{j-q_{i-1}}\left(S^{(i)}\right) a_{q_{i}}(S) \chi\left(S_{q_{i}-1}, S_{q_{i}}\right),
\end{array}\right.
$$

for $q_{i-1} \leq j<q_{i}$, to be used with decreasing $i=l(S)-2, l(S)-3, \ldots, 1$, and with clarification as follows. Since $t(S)>0$, we must have $l(S) \geq 2$. If $l(S)=2$, then, upon letting $\nu^{\prime}=t(S)$ and

$$
S^{\prime}=\left(S_{j}, x_{j}, y_{j}, \kappa_{j}\right)_{0 \leq j \leq \nu^{\prime}},
$$

we have that $S^{\prime}$ is a nonflip with $\nu^{\prime}>0$, and hence by previous constructions the positive integers

$$
m_{j}^{\prime}\left(S^{\prime}\right), a_{j}^{\prime}\left(S^{\prime}\right), b_{j}^{\prime}\left(S^{\prime}\right)
$$

are defined for $0 \leq j \leq \nu^{\prime}-1$, and hence, without applying the recurrence formulas, we are done by taking

$$
\left(m_{j}(S), a_{j}(S), b_{j}(S)\right)=\left(m_{j}^{\prime}\left(S^{\prime}\right), a_{j}^{\prime}\left(S^{\prime}\right), b_{j}^{\prime}\left(S^{\prime}\right)\right) \text { for } 0 \leq j \leq \nu^{\prime}-1 .
$$

So now assume that $l(S)>2$. Then upon letting $\nu^{\prime}=t(S)-q_{l(S)-2}$ and

$$
S^{\prime}=\left(S_{q_{l(S)-2}+j}, x_{q_{l(S)-2}+j}, y_{q_{l(S)-2}+j}, \kappa_{q_{l(S)-2}+j}\right)_{0 \leq j \leq \nu^{\prime}},
$$

we have that $S^{\prime}$ is either a flip or a nonflip with $\nu^{\prime}>0$, and hence by previous constructions the positive integers

$$
m_{j}^{\prime}\left(S^{\prime}\right), a_{j}^{\prime}\left(S^{\prime}\right), b_{j}^{\prime}\left(S^{\prime}\right)
$$

are defined for $0 \leq j \leq \nu^{\prime}-1$, and we take them to be the values of

$$
m_{j-q_{l(S)-2}}(S), a_{j-q_{l(S)-2}}(S), b_{j-q_{l(S)-2}}(S)
$$

for $q_{l(S)-2} \leq j<q_{l(S)-1}$ respectively. Now use $\left(r_{i}\right)$ for $i=l(S)-2, l(S)-3, \ldots, 1$.

(2D) Properties. Consider a sprout on $S$, which is a sequence $\left(F_{j}, I_{j}, d_{j}\right)_{0 \leq j \leq \nu}$ where $0 \neq F_{j} \in M\left(S_{j}\right)$ with $I_{j}=F_{j} S_{j}$ and $d_{j}=\operatorname{ord}_{S_{j}} I_{j}$ such that for $0 \leq j<\nu$ we have

$$
F_{j} / F_{j+1}=z_{j}^{d_{j}} \text { with } z_{j}= \begin{cases}x_{j} & \text { if } x_{j+1}=x_{j} \\ y_{j} & \text { if } y_{j+1}=y_{j}\end{cases}
$$

and $\left(S_{j}, S_{j+1}^{\prime}\right)\left(I_{j}\right)=S_{j+1}^{\prime}$ whenever $S_{j+1}^{\prime} \in Q_{1}\left(S_{j}\right) \backslash\left\{S_{j+1}\right\}$.

In the next section, as a consequence of the spider principle, we shall show the following:

Lemma 2.1. Assume that $S$ is either a flip or a nonflip with $\nu>0$. Then

$$
d_{j}=m_{j}(S) d_{\nu-1} \text { for } 0 \leq j \leq \nu-1 .
$$

Moreover, for $0 \leq j \leq \nu-1$, we have $m_{j}(S)=\min \left(a_{j}(S), b_{j}(S)\right)$ and, in the terminology to be introduced in the next section, the Newton polygon $N\left(F_{j}\right)$ is a triangle with top point $\left(0, a_{j}(S) d_{\nu-1}\right)$ and bottom point $\left(b_{j}(S) d_{\nu-1}, 0\right)$. 
Theorem 2.2. Assume $t(S)>0$. Then

$$
d_{j}=m_{j}(S) d_{\nu-1} \text { for } 0 \leq j \leq t(S)-1 .
$$

Moreover, for $0 \leq j \leq t(S)-1$, we have $m_{j}(S)=\min \left(a_{j}(S), b_{j}(S)\right)$ and, in the terminology to be introduced in the next section, the Newton polygon $N\left(F_{j}\right)$ is a triangle with top point $\left(0, a_{j}(S) d_{t(S)-1}\right)$ and bottom point $\left(b_{j}(S) d_{t(S)-1}, 0\right)$.

\section{NeWTON POLYGON}

The most relevant notation for this section is the notation about the Newton Polygon introduced in our previous paper [AL1], which the reader should have by his side.

Let $R$ be a two dimensional regular local domain with quotient field $\Omega$, residue field $K=H(R)$, generators $(x, y)$ of $M(R)$, and coefficient set $\kappa$ of $R$.

To avoid confusion with the $S$ of the previous section, let us put $\sharp$ on top of the $S$ occurring in [AL1]. In $(3)(\dagger)$ of [AL1, for any

$$
\emptyset \neq S^{\sharp} \subset \mathbb{N}^{2}=\{(\Lambda, L): \Lambda \in \mathbb{N}, L \in \mathbb{N}\}
$$

we have defined

$$
\mathcal{N}\left(S^{\sharp}\right)=E\left(S^{\sharp}\right) \amalg C\left(S^{\sharp}\right),
$$

where $\mathcal{N}\left(S^{\sharp}\right), E\left(S^{\sharp}\right), C\left(S^{\sharp}\right)$ are called the Newton polygon, the edge set, and the corner set of $S^{\sharp}$ respectively, and where the corners of the polygon, i.e., the points of $C\left(S^{\sharp}\right)$, are labelled as $P_{1}=\left(\Lambda_{1}, L_{1}\right), \ldots, P_{\iota+1}=\left(\Lambda_{\iota+1}, L_{\iota+1}\right)$ where $\mathcal{N}\left(S^{\sharp}\right)=$ $\left\{P_{1}\right\} \Leftrightarrow P_{1}=P_{\iota+1} \Leftrightarrow \iota=0$. Moreover, if $\iota \neq 0$, then the points $P_{1}, \ldots, P_{\iota+1}$ are pairwise distinct and the cardinality $\left|C\left(S^{\sharp}\right)\right|=\iota+1$ with $\iota \in \mathbb{N}_{+}$, the sides of the polygon are the segments $Q_{1}, \ldots, Q_{\iota}$ where $Q_{i}$ joins $P_{i}$ to $P_{i+1}$, and the slopes of $Q_{1}, \ldots, Q_{\iota}$ are the positive rational numbers $O_{1}<\cdots<O_{\iota}$. We put $\iota\left(\mathcal{N}\left(S^{\sharp}\right)\right)=\iota=$ the index of $\mathcal{N}\left(S^{\sharp}\right)$. We call $\mathcal{N}\left(S^{\sharp}\right)$ a triangle if $\iota=1$ with $\Lambda_{1}=0 \neq L_{1}$ and $\Lambda_{2} \neq 0=L_{2}$; we call $\left(0, L_{1}\right)$ and $\left(\Lambda_{2}, 0\right)$ the top point and the bottom point of the triangle respectively; by the slope of the triangle we mean the slope of the segment joining $\left(0, L_{1}\right)$ to $\left(\Lambda_{2}, 0\right)$, i.e., the positive rational number $\Lambda_{2} / L_{1}$.

As stated at the beginning of Section 4 of AL1, relative to $(R, x, y, \kappa)$, any element $F$ of $R$ has a unique Taylor expansion

$$
F=\sum_{(i, j) \in \mathbb{N}^{2}} \bar{F}_{i j} x^{i} y^{j}
$$

in the completion $\widehat{R}$ of $R$ with $\bar{F}_{i j} \in \kappa$, and by $S^{\sharp}(R, x, y, \kappa)(F)$ we denote the support of $F$ relative to $(R, x, y, \kappa)$, i.e.,

$$
S^{\sharp}(R, x, y, \kappa)(F)=\left\{(i, j) \in \mathbb{N}^{2}: \bar{F}_{i j} \neq 0\right\},
$$

and we note that if $F \neq 0$, then its support is nonempty. For brevity, we may refer to $\mathcal{N}\left(S^{\sharp}(R, x, y, \kappa)(F)\right)$ as the Newton polygon of $F$ relative to $(R, x, y, \kappa)$ and denote it by $N(F)$. Usually we shall call $N(F)$ the Newton polygon of $F$ without explicitly mentioning $(R, x, y, \kappa)$. If the index $\iota(N(F) \neq 0$, then the sides $Q_{1}, \ldots, Q_{\iota}$ and corners $P_{1}, \ldots, P_{\iota+1}$ may be called the (first, second, ... , last) Newton lines and the (first, second, ... , last) Newton points of $F$ respectively. If $\iota(N(F))=0$, then $N(F)$ is reduced to the single point $P_{1}=P_{\iota+1}$. 
For the finite QDT sequence $S=\left(S_{j}, x_{j}, y_{j}, \kappa_{j}\right)_{0 \leq j \leq \nu}$ of $(R, x, y, \kappa)$ and the sprout $\left(F_{j}, I_{j}, d_{j}\right)_{0 \leq j \leq \nu}$ on $S$ described at the end of the last section, we are going to consider the Newton polygon $N\left(F_{j}\right)$ of $F_{j}$ relative to $\left(S_{j}, x_{j}, y_{j}, \kappa_{j}\right)$ for $0 \leq j \leq \nu$.

Lemma 3.1. Assuming $\nu>0$ we have the following.

(3.1.1). Assume that $\iota\left(N\left(F_{0}\right)\right)=0$; i.e., $N\left(F_{0}\right)$ is reduced to a single point. Then that point is either $\left(0, d_{0}\right)$ or $\left(d_{0}, 0\right)$. In the first case, 1 is an $X$-operation of $S$, and $N\left(F_{1}\right)$ is reduced to the single point $\left(0, d_{1}\right)=\left(0, d_{0}\right)$. In the second case, 1 is a $Y$-operation of $S$, and $N\left(F_{1}\right)$ is reduced to the single point $\left(d_{1}, 0\right)=\left(d_{0}, 0\right)$.

(3.1.2). Assume that $N\left(F_{0}\right)$ is a triangle with top point $\left(0, a_{0}\right)$ and bottom point $\left(b_{0}, 0\right)$, and hence with slope $b_{0} / a_{0}$. Then $d_{0}=\min \left(a_{0}, b_{0}\right)$. Moreover we have $a_{0}<b_{0}$ or $a_{0}>b_{0}$ or $a_{0}=b_{0}$ according as 1 is an $X$-operation or $Y$-operation or a translation of $S$.

(3.1.3). 1 is an $X$-operation of $S$ iff the first Newton point of $F_{0}$ is $\left(0, d_{0}\right)$ and either $\iota\left(N\left(F_{0}\right)\right)=0$ and this is the only Newton point of $F_{0}$ or $\iota\left(N\left(F_{0}\right)\right)>0$ and the first Newton line of $F_{0}$ has slope $>1$. Moreover, if 1 is an $X$-operation of $S$, then the first Newton point of $F_{1}$ is of the form $\left(0, a_{1}\right)$ with $a_{1} \in \mathbb{N}_{+}$and we have $d_{0}=\chi\left(S_{0}, S_{1}\right) a_{1}=a_{1}$.

(3.1.4). 1 is a $Y$-operation of $S$ iff the last Newton point of $F_{0}$ is $\left(d_{0}, 0\right)$ and either $\iota\left(N\left(F_{0}\right)\right)=0$ and this is the only Newton point of $F_{0}$ or $\iota\left(N\left(F_{0}\right)\right)>0$ and the last Newton line of $F_{0}$ has slope $<1$. Moreover, if 1 is a $Y$-operation of $S$, then the last Newton point of $F_{1}$ is of the form $\left(b_{1}, 0\right)$ with $b_{1} \in \mathbb{N}_{+}$and we have $d_{0}=\chi\left(S_{0}, S_{1}\right) b_{1}=b_{1}$.

(3.1.5). 1 is a translation of $S$ iff $N\left(F_{0}\right)$ is a triangle of slope 1 . Moreover, if 1 is a translation of $S$, then the first Newton point of $F_{1}$ is of the form $\left(0, a_{1}\right)$ with $a_{1} \in \mathbb{N}_{+}$and we have $d_{0}=\chi\left(S_{0}, S_{1}\right) a_{1}$.

(3.1.6). Assume that 1 is a translation of $S$ and $N\left(F_{1}\right)$ is a triangle with top point $\left(0, a_{1}\right)$ and bottom point $\left(b_{1}, 0\right)$. Then $N\left(F_{0}\right)$ is a triangle with top point $\left(0, \chi\left(S_{0}, S_{1}\right) a_{1}\right)$ and bottom point $\left(\chi\left(S_{0}, S_{1}\right) a_{1}, 0\right)$.

(3.1.7). Assume that 1 is an $X$-operation of $S$ and $N\left(F_{1}\right)$ is a triangle with top point $\left(0, a_{1}\right)$ and bottom point $\left(b_{1}, 0\right)$. Then $\chi\left(S_{0}, S_{1}\right)=1$ and $N\left(F_{0}\right)$ is a triangle with top point $\left(0, a_{0}\right)=\left(0, a_{1}\right)$ and bottom point $\left(b_{0}, 0\right)=\left(a_{1}+b_{1}, 0\right)$.

(3.1.8). Assume that 1 is a $Y$-operation of $S$ and $N\left(F_{1}\right)$ is a triangle with top point $\left(0, a_{1}\right)$ and bottom point $\left(b_{1}, 0\right)$. Then $\chi\left(S_{0}, S_{1}\right)=1$ and $N\left(F_{0}\right)$ is a triangle with top point $\left(0, a_{0}\right)=\left(0, a_{1}+b_{1}\right)$ and bottom point $\left(b_{0}, 0\right)=\left(b_{1}, 0\right)$.

Proof of (3.1). (3.1.1) to (3.1.5) follow from (2A) to (2C) of Ab13. By (3.1.5) we get (3.1.6). Invoking Lemma 3.1 of [AL1, by (3.1.1), (3.1.2), and (3.1.3) we get (3.1.7). Invoking Lemma 3.2 of [AL1], by (3.1.1), (3.1.2), and (3.1.4) we get (3.1.8).

Proof of (2.1). Briefly speaking, the nonflip case follows from (3.1.1) to (3.1.7) except (3.1.4), whereas the flip case follows from (3.1.1) to (3.1.8). In greater detail, i.e., in the Sanskrit tradition, after the MANTRA there is the BHASHYA.

First consider the case when $S$ is a nonflip with $\nu>0$. Now clearly $t(S)=\nu>0$ with $l(S)=2$, and hence by definition

$$
\left(m_{j}(S), a_{j}(S), b_{j}(S)\right)=\left(m_{j}^{\prime}(S), a_{j}^{\prime}(S), b_{j}^{\prime}(S)\right) \text { for } 0 \leq j \leq \nu-1 .
$$

By the nonflip part of the definition of $m^{\prime}$ and part (ii) of the definition of $a^{\prime}, b^{\prime}$, we see that $m_{\nu-1}^{\prime}(S)=a_{\nu-1}^{\prime}(S)=b_{\nu-1}^{\prime}(S)=1$ and hence

$$
m_{\nu-1}(S)=a_{\nu-1}(S)=b_{\nu-1}(S)=1 \text {. }
$$


By the definition of a nonflip, $\nu$ is a translation of $S$, and hence by (3.1.2) together with the first sentence of (3.1.5) we see that $N\left(F_{\nu-1}\right)$ is a triangle with top point $\left(0, d_{\nu-1}\right)$ and bottom point $\left(d_{\nu-1}, 0\right)$. Consequently, by $(2)$ we conclude that for $j=\nu-1$ we have $d_{j}=m_{j}(S) d_{\nu-1}$ with $m_{j}(S)=\min \left(a_{j}(S), b_{j}(S)\right)$ and the Newton polygon $N\left(F_{j}\right)$ is a triangle with top point $\left(0, a_{j}(S) d_{\nu-1}\right)$ and bottom point $\left(b_{j}(S) d_{\nu-1}, 0\right)$. Now, in view of (1) and the nonflip part of the definition of $m^{\prime}$, together with part (ii) of the definition of $a^{\prime}, b^{\prime}$, and invoking (3.1.1) to (3.1.7) except (3.1.4), by decreasing induction on $j$ we see that for $0 \leq j<\nu-1$ we have $d_{j}=m_{j}(S) d_{\nu-1}$ with $m_{j}(S)=\min \left(a_{j}(S), b_{j}(S)\right)$ and the Newton polygon $N\left(F_{j}\right)$ is a triangle with top point $\left(0, a_{j}(S) d_{\nu-1}\right)$ and bottom point $\left(b_{j}(S) d_{\nu-1}, 0\right)$.

Next consider the case when $S$ is a flip. Now $t(S)=\nu=q_{2}(S) \geq 2$ with $l(S)=3=3+q_{1}(S)$, and hence by definition we have (1). By the definition of $m^{\prime}, a^{\prime}, b^{\prime}$ we see that $m_{\nu-1}^{\prime}(S)=a_{\nu-1}^{\prime}(S)=b_{\nu-1}^{\prime}(S)=1$, and hence we get $(2)$. By the definition of a flip, $\nu$ is a translation of $S$, and hence by (3.1.2) together with the first sentence of (3.1.5) we see that $N\left(F_{\nu-1}\right)$ is a triangle with top point $\left(0, d_{\nu-1}\right)$ and bottom point $\left(d_{\nu-1}, 0\right)$. Consequently, by (2) we conclude that for $j=\nu-1$ we have $d_{j}=m_{j}(S) d_{\nu-1}$ with $m_{j}(S)=\min \left(a_{j}(S), b_{j}(S)\right)$ and the Newton polygon $N\left(F_{j}\right)$ is a triangle with top point $\left(0, a_{j}(S) d_{\nu-1}\right)$ and bottom point $\left(b_{j}(S) d_{\nu-1}, 0\right)$. Now, in view of (1) together with the definition of $m^{\prime}, a^{\prime}, b^{\prime}$, and invoking (3.1.1) to (3.1.8), by decreasing induction on $j$ we see that for $0 \leq j<\nu-1$ we have $d_{j}=m_{j}(S) d_{\nu-1}$ with $m_{j}(S)=\min \left(a_{j}(S), b_{j}(S)\right)$ and the Newton polygon $N\left(F_{j}\right)$ is a triangle with top point $\left(0, a_{j}(S) d_{\nu-1}\right)$ and bottom point $\left(b_{j}(S) d_{\nu-1}, 0\right)$.

Proof of (2.2). Referring to the recurrence formulas $\left(r_{i}\right)$ of Section 2, the general case of $t(S)>0$ follows from (2.1) by decreasing induction on $i$.

\section{Head multiplicity Sequence}

Let $R$ be a two dimensional regular local domain with quotient field $\Omega$, residue field $K=H(R)$, generators $(x, y)$ of $M(R)$, and coefficient set $\kappa$ of $R$. As in Section 2 , let $S=\left(S_{j}, x_{j}, y_{j}, \kappa_{j}\right)_{0 \leq j \leq \nu}$ be a finite QDT sequence of $(R, x, y, \kappa)$. We shall now extend the multiplicity sequence to include the head $S^{(l(S))}$ of $S$.

Definition 4.1. Given any positive integers $a, b$ with $\operatorname{GCD}(a, b)=1$, we introduce a triple sequence $\left(m_{j}^{*}, a_{j}^{*}, b_{j}^{*}\right)_{0 \leq j \leq \nu}$ of positive integers by the initial condition

$$
\left(m_{\nu}^{*}, a_{\nu}^{*}, b_{\nu}^{*}\right)=(\min (a, b), a, b)
$$

followed by the recurrence formula

$$
\left(m_{j}^{*}, a_{j}^{*}, b_{j}^{*}\right)= \begin{cases}\left(a_{j+1}^{*}, a_{j+1}^{*}, a_{j+1}^{*}+b_{j+1}^{*}\right) & \text { if } j+1 \text { is an } X \text {-operation } \\ \left(b_{j+1}^{*}, a_{j+1}^{*}+b_{j+1}^{*}, b_{j+1}^{*}\right) & \text { if } j+1 \text { is a } Y \text {-operation }\end{cases}
$$

for $t(S) \leq j<\nu$ with decreasing induction on $j$ and ending with the formula

$$
\left(m_{j}^{*}, a_{j}^{*}, b_{j}^{*}\right)=\left(m_{j}(S) m_{t(S)}^{*}, a_{j}(S) m_{t(S)}^{*}, b_{j}(S) m_{t(S)}^{*}\right)
$$

for $0 \leq j<t(S)$. We define the supermultiplicity sequence

$$
m^{*}(S, a, b)=m_{j}^{*}(S, a, b)_{0 \leq j \leq \nu}
$$

together with the superglue bisequence

$$
\left(a^{*}(S, a, b), b^{*}(S, a, b)\right)=\left(a_{j}^{*}(S, a, b), b_{j}^{*}(S, a, b)\right)_{0 \leq j \leq \nu}
$$


of $(S, a, b)$ by putting

$$
\left(m_{j}^{*}(S, a, b), a_{j}^{*}(S, a, b), b_{j}^{*}(S, a, b)\right)=\left(m_{j}^{*}, a_{j}^{*}, b_{j}^{*}\right) \text { for } 0 \leq j \leq \nu .
$$

We define the submultiplicity sequence

$$
m^{* *}(S)=m_{j}^{* *}(S)_{0 \leq j \leq \nu}
$$

together with the subglue bisequence

$$
\left(a^{* *}(S), b^{* *}(S)\right)=\left(a_{j}^{* *}(S), b_{j}^{* *}(S)\right)_{0 \leq j \leq \nu}
$$

of $S$ by putting

$$
\left(m_{j}^{* *}(S), a_{j}^{* *}(S), b_{j}^{* *}(S)\right)= \begin{cases}(1,1,1) & \text { if } t(S) \leq j \leq \nu \\ \left(m_{j}(S), a_{j}(S), b_{j}(S)\right) & \text { if } 0 \leq j<t(S) .\end{cases}
$$

Theorem 4.2. For the sprout $\left(F_{j}, I_{j}, d_{j}\right)_{0 \leq j \leq \nu}$ on $S$ described at the end of Section 2, considering the Newton polygon $N\left(F_{j}\right)$ of $F_{j}$ relative to $\left(S_{j}, x_{j}, y_{j}, \kappa_{j}\right)$ for $0 \leq j \leq \nu$, we have the following.

(4.2.1) Assume that $N\left(F_{\nu}\right)$ is a triangle with top point $(0, A)$ and bottom point $(B, 0)$. Let $D=G C D(A, B)$ and let $(a, b)=(A / D, B / D)$. Then, for $0 \leq j \leq \nu$, upon letting

we have

$$
\left(m_{j}^{*}, a_{j}^{*}, b_{j}^{*}\right)=\left(m_{j}^{*}(S, a, b), a_{j}^{*}(S, a, b), b_{j}^{*}(S, a, b)\right),
$$

$$
d_{j}=m_{j}^{*} D=\min \left(a_{j}^{*} D, b_{j}^{*} D\right)
$$

and $N\left(F_{j}\right)$ is a triangle with top point $\left(0, a_{j}^{*} D\right)$ and bottom point $\left(b_{j}^{*} D, 0\right)$.

(4.2.2) Assume that the first Newton point of $F_{\nu}$ is $\left(0, d_{\nu}\right)$ and for every integer $j$ with $t(S)<j \leq \nu$ we have that $j$ is an $X$-operation of $S$. Then: (1) for $0 \leq j \leq \nu$ we have $d_{j}=m_{j}^{* *}(S) d_{\nu}$, (2) for $t(S) \leq j \leq \nu$ we have that the first Newton point of $F_{j}$ is $\left(0, d_{j}\right)=\left(0, d_{\nu}\right)$, and (3) for $0 \leq j<t(S)$ we have that $N\left(F_{j}\right)$ is a triangle with top point $\left(0, a_{j}^{* *}\right)$ and bottom point $\left(b_{j}^{* *}, 0\right)$.

(4.2.3) Assume that the last Newton point of $F_{\nu}$ is $\left(d_{\nu}, 0\right)$ and for every integer $j$ with $t(S)<j \leq \nu$ we have that $j$ is a $Y$-operation of $S$. Then: (1) for $0 \leq j \leq \nu$ we have $d_{j}=m_{j}^{* *}(S) d_{\nu}$, (2) for $t(S) \leq j \leq \nu$ we have that the last Newton point of $F_{j}$ is $\left(d_{j}, 0\right)=\left(d_{\nu}, 0\right)$, and (3) for $0 \leq j<t(S)$ we have that $N\left(F_{j}\right)$ is a triangle with top point $\left(0, a_{j}^{* *}\right)$ and bottom point $\left(b_{j}^{* *}, 0\right)$.

(4.2.4) Assume that $N\left(F_{\nu}\right)$ is reduced to a single point of the form $(0, A)$ with $A \in \mathbb{N}_{+}$. Then for every integer $j$ with $t(S)<j \leq \nu$ we have that $j$ is an $X$ operation of $S$ and $N\left(F_{j-1}\right)$ is reduced to the single point $(0, A)$. Moreover, for $t(S) \leq j \leq \nu$ we have $d_{j}=A$.

(4.2.5) Assume that $N\left(F_{\nu}\right)$ is reduced to a single point of the form $(B, 0)$ with $B \in \mathbb{N}_{+}$. Then for every integer $j$ with $t(S)<j \leq \nu$ we have that $j$ is a $Y$-operation of $S$ and $N\left(F_{j-1}\right)$ is reduced to the single point $(B, 0)$. Moreover, for $t(S) \leq j \leq \nu$ we have $d_{j}=B$.

Proof of (4.2.1). Follows from (2.2), (3.1.7), and (3.1.8).

Proof of (4.2.2). Follows from (2.2) and (3.1.3).

Proof of (4.2.3). Follows from (2.2) and (3.1.4).

Proof of (4.2.4). Follows from (3.1.1) to (3.1.4).

Proof of (4.2.5). Follows from (3.1.1) to (3.1.4). 
Definition 4.3. Let $P(R)$ denote the set of all nonzero principal ideals in $R$, and let $P^{\times}(R)=P(R) \backslash\{R\}$ and $P^{*}(R)=P(R) \cap \operatorname{spec}(R)$. Note that if $V=o\left(S_{\nu}\right)$, then $o_{R}^{-1}(V)=S_{\nu}$; by analogy, for any $T \in Q_{1}\left(S_{\nu}\right)$ we put

$$
o_{R}^{*}(T)=S_{\nu},
$$

and we put

$$
\pi(R, V)=Q_{1}\left(S_{\nu}\right)=Q_{1}\left(o_{R}^{-1}(V)\right)=\text { set of all points of } V
$$

and

$$
\left\{\begin{aligned}
\pi^{*}(R, V) & =\left\{T \in \pi(R, V): T \not \subset o\left(S_{j}\right) \text { for } 0 \leq j<\nu\right\} \\
& =\text { set of all free points of } V .
\end{aligned}\right.
$$

For any $T \in \pi(R, V)$ with $V \in D(R)^{\Delta}$ we put

$$
\xi(R, T, V)=T \cap M(V)=\text { the parameter ideal of }(R, T, V),
$$

and we note that then we have $\xi(R, T, V) \in P^{*}(T)$ with $\operatorname{ord}_{T} \xi(R, T, V)=1$ and $V=T_{\xi(R, T, V)}$. We put

$$
\gamma(R, T, V)=\left\{\begin{array}{l}
\left\{\mathfrak{c} \in P^{\times}(T): \operatorname{ord}_{T} \mathfrak{c}=\operatorname{ord}_{(T / \xi(R, T, V))} \mathfrak{c}\right\} \\
=\text { set of all arcs of }(R, T, V)
\end{array}\right.
$$

and we define the weight of $\mathfrak{c}$ by letting

$$
\theta(R, T, \mathfrak{c})=\chi\left(o_{R}^{*}(T), T\right) \operatorname{ord}_{T} \mathfrak{c},
$$

and we put

$$
\gamma^{*}(R, T, V)=\left\{\begin{array}{l}
\gamma(R, T, V) \text { or } \emptyset \text { according as } T \in \pi^{*}(R, V) \text { or } T \notin \pi^{*}(R, V) \\
=\text { set of all curvettes of }(R, T, V) .
\end{array}\right.
$$

Lemma 4.4. Assuming $\nu>0$ we have the following.

(4.4.1). Assume that $x_{\nu}=x_{\nu-1}$ and let $T$ be the unique member of $Q_{1}\left(S_{\nu}\right)$ with $M(T)=\left(x_{\nu} / y_{\nu}, y_{\nu}\right) T$. Then $T \subset o\left(S_{\nu-1}\right)$ and for all $T^{*} \in Q_{1}\left(S_{\nu}\right) \backslash\{T\}$ we have $T^{*} \notin o\left(S_{\nu-1}\right)$.

(4.4.2). Assume that $y_{\nu}=y_{\nu-1}$. Let $U=o\left(S_{\nu-1}\right)$ and let $T$ be the unique member of $Q_{1}\left(S_{\nu}\right)$ with $M(T)=\left(x_{\nu}, y_{\nu} / x_{\nu}\right) T$. Then $T \subset o\left(S_{\nu-1}\right)$ and for all $T^{*} \in Q_{1}\left(S_{\nu}\right) \backslash\{T\}$ we have $T^{*} \notin o\left(S_{\nu-1}\right)$.

(4.4.3). Assume that $\nu>\nu^{\prime}>0$ for an integer $\nu^{\prime}$ such that $y_{\nu^{\prime}}=y_{\nu^{\prime}-1}$ and $\bar{T} \subset o\left(S_{\nu^{\prime}-1}\right)$ for some $\bar{T} \in Q_{1}\left(S_{\nu}\right)$. Then for every integer $j$ with $\nu^{\prime}<j \leq \nu$ we have $y_{j}=y_{j-1} / x_{j-1}$, and letting $T^{\prime}$ be the unique member of $Q_{1}\left(S_{\nu}\right)$ with $M\left(T^{\prime}\right)=\left(x_{\nu}, y_{\nu} / x_{\nu}\right) T^{\prime}$, we have $T^{\prime} \subset o\left(S_{\nu^{\prime}-1}\right)$ and for all $T^{*} \in Q_{1}\left(S_{\nu}\right) \backslash\left\{T^{\prime}\right\}$ we have $T^{*} \notin o\left(S_{\nu^{\prime}-1}\right)$.

(4.4.4). Assume that $\nu>\nu^{\prime}>0$ for an integer $\nu^{\prime}$ such that $x_{\nu^{\prime}}=x_{\nu^{\prime}-1}$ and $\bar{T} \subset o\left(S_{\nu^{\prime}-1}\right)$ for some $\bar{T} \in Q_{1}\left(S_{\nu}\right)$. Then for every integer $j$ with $\nu^{\prime}<j \leq \nu$ we have $x_{j}=x_{j-1} / y_{j-1}$, and letting $T^{\prime}$ be the unique member of $Q_{1}\left(S_{\nu}\right)$ with $M\left(T^{\prime}\right)=\left(x_{\nu} / y_{\nu}, y_{\nu}\right) T^{\prime}$, we have $T^{\prime} \subset o\left(S_{\nu^{\prime}-1}\right)$ and for all $T^{*} \in Q_{1}\left(S_{\nu}\right) \backslash\left\{T^{\prime}\right\}$ we have $T^{*} \notin o\left(S_{\nu^{\prime}-1}\right)$.

(4.4.5). Assume that $\nu>\nu^{\prime}>0$ for an integer $\nu^{\prime}$ such that $y_{\nu^{\prime}}=y_{\nu^{\prime}-1}$ and for every integer $j$ with $\nu^{\prime}<j \leq \nu$ we have $y_{j}=y_{j-1} / x_{j-1}$. Then, letting $T^{\prime}$ be the unique member of $Q_{1}\left(S_{\nu}\right)$ with $M\left(T^{\prime}\right)=\left(x_{\nu}, y_{\nu} / x_{\nu}\right) T^{\prime}$, we have $T^{\prime} \subset o\left(S_{\nu^{\prime}-1}\right)$ and for all $T^{*} \in Q_{1}\left(S_{\nu}\right) \backslash\left\{T^{\prime}\right\}$ we have $T^{*} \notin o\left(S_{\nu^{\prime}-1}\right)$. 
(4.4.6). Assume that $\nu>\nu^{\prime}>0$ for an integer $\nu^{\prime}$ such that $x_{\nu^{\prime}}=x_{\nu^{\prime}-1}$ and for every integer $j$ with $\nu^{\prime}<j \leq \nu$ we have $x_{j}=x_{j-1} / y_{j-1}$. Then, letting $T^{\prime}$ be the unique member of $Q_{1}\left(S_{\nu}\right)$ with $M\left(T^{\prime}\right)=\left(x_{\nu} / y_{\nu}, y_{\nu}\right) T^{\prime}$, we have $T^{\prime} \subset o\left(S_{\nu^{\prime}-1}\right)$ and for all $T^{*} \in Q_{1}\left(S_{\nu}\right) \backslash\left\{T^{\prime}\right\}$ we have $T^{*} \notin o\left(S_{\nu^{\prime}-1}\right)$.

Note. In the following proofs we shall tacitly use the fact that for any positive integer $j \leq \nu$ we have that $j$ is a translation of $S$ iff $y_{j-1} / x_{j-1}$ is a unit in $S_{j}$.

Proof of (4.4.1). Let $U=o\left(S_{\nu-1}\right)$. Then $S_{\nu} \subset U$ with $\operatorname{ord}_{U} x_{\nu}=1$ and $\operatorname{ord}_{U} y_{\nu}=$ 0 . Consequently $T \subset U$ and for all $T^{*} \in Q_{1}\left(S_{\nu}\right) \backslash\{T\}$ we have $T^{*} \notin U$.

Proof of (4.4.2). Let $U=o\left(S_{\nu-1}\right)$. Then $S_{\nu} \subset U$ with $\operatorname{ord}_{U} y_{\nu}=1$ and $\operatorname{ord}_{U} x_{\nu}=$ 0 . Consequently $T \subset U$ and for all $T^{*} \in Q_{1}\left(S_{\nu}\right) \backslash\{T\}$ we have $T^{*} \notin U$.

Proof of (4.4.3). Let $U^{\prime}=o\left(S_{\nu^{\prime}-1}\right)$. Now

$$
\left\{\begin{array}{l}
S_{\nu^{\prime}} \subset S_{\nu^{\prime}+1} \subset S_{\nu} \subset \bar{T} \subset U^{\prime} \text { and } U^{\prime}=o\left(S_{\nu^{\prime}-1}\right) \text { with } y_{\nu^{\prime}}=y_{\nu^{\prime}-1} \\
\Rightarrow\left(\operatorname{ord}_{U^{\prime}} x_{\nu^{\prime}}, \operatorname{ord}_{U^{\prime}} y_{\nu^{\prime}}\right)=(0,1) \\
\Rightarrow\left(x_{\nu^{\prime}+1}, y_{\nu^{\prime}+1}\right)=\left(x_{\nu^{\prime}}, y_{\nu^{\prime}} / x_{\nu^{\prime}}\right) \text { with }\left(\operatorname{ord}_{U^{\prime}} x_{\nu^{\prime}+1}, \operatorname{ord}_{U^{\prime}} y_{\nu^{\prime}+1}\right)=(0,1),
\end{array}\right.
$$

and if $\nu^{\prime}+1<\nu$, then

$$
\left\{\begin{array}{l}
S_{\nu^{\prime}+1} \subset S_{\nu^{\prime}+2} \subset S_{\nu} \subset \bar{T} \subset U^{\prime} \text { with }\left(\operatorname{ord}_{U^{\prime}} x_{\nu^{\prime}+1}, \operatorname{ord}_{U^{\prime}} y_{\nu^{\prime}+1}\right)=(0,1) \\
\Rightarrow\left(x_{\nu^{\prime}+2}, y_{\nu^{\prime}+2}\right)=\left(x_{\nu^{\prime}+1}, y_{\nu^{\prime}+1} / x_{\nu^{\prime}+1}\right) \text { with }\left(\operatorname{ord}_{U^{\prime}} x_{\nu^{\prime}+2}, \operatorname{ord}_{U^{\prime}} y_{\nu^{\prime}+2}\right)=(0,1),
\end{array}\right.
$$

and if $\nu^{\prime}+2<\nu$, then and so on. Thus by induction on $j$ we see that for $\nu^{\prime}<j \leq \nu$ we have

$$
\left(x_{j}, y_{j}\right)=\left(x_{j-1}, y_{j-1} / x_{j-1}\right) \text { with }\left(\operatorname{ord}_{U^{\prime}} x_{j}, \operatorname{ord}_{U^{\prime}} y_{j}\right)=(0,1) .
$$

Hence, letting $T^{\prime}$ be the unique member of $Q_{1}\left(S_{\nu}\right)$ with $M\left(T^{\prime}\right)=\left(x_{\nu}, y_{\nu} / x_{\nu}\right) T^{\prime}$, we see that $T^{\prime} \subset U^{\prime}$ and for all $T^{*} \in Q_{1}\left(S_{\nu}\right) \backslash\left\{T^{\prime}\right\}$ we have $T^{*} \notin U^{\prime}$.

Proof of (4.4.4). Let $U^{\prime}=o\left(S_{\nu^{\prime}-1}\right)$. Now

$$
\left\{\begin{array}{l}
S_{\nu^{\prime}} \subset S_{\nu^{\prime}+1} \subset S_{\nu} \subset \bar{T} \subset U^{\prime} \text { and } U^{\prime}=o\left(S_{\nu^{\prime}-1}\right) \text { with } x_{\nu^{\prime}}=x_{\nu^{\prime}-1} \\
\Rightarrow\left(\operatorname{ord}_{U^{\prime}} x_{\nu^{\prime}}, \operatorname{ord}_{U^{\prime}} y_{\nu^{\prime}}\right)=(1,0) \\
\Rightarrow\left(x_{\nu^{\prime}+1}, y_{\nu^{\prime}+1}\right)=\left(x_{\nu^{\prime}} / y_{\nu^{\prime}}, y_{\nu^{\prime}}\right) \text { with }\left(\operatorname{ord}_{U^{\prime}} x_{\nu^{\prime}+1}, \operatorname{ord}_{U^{\prime}} y_{\nu^{\prime}+1}\right)=(1,0),
\end{array}\right.
$$

and if $\nu^{\prime}+1<\nu$, then

$$
\left\{\begin{array}{l}
S_{\nu^{\prime}+1} \subset S_{\nu^{\prime}+2} \subset S_{\nu} \subset \bar{T} \subset U^{\prime} \text { with }\left(\operatorname{ord}_{U^{\prime}} x_{\nu^{\prime}+1}, \operatorname{ord}_{U^{\prime}} y_{\nu^{\prime}+1}\right)=(1,0) \\
\Rightarrow\left(x_{\nu^{\prime}+2}, y_{\nu^{\prime}+2}\right)=\left(x_{\nu^{\prime}+1} / y_{\nu^{\prime}+1}, y_{\nu^{\prime}+1}\right) \text { with }\left(\operatorname{ord}_{U^{\prime}} x_{\nu^{\prime}+2}, \operatorname{ord}_{U^{\prime}} y_{\nu^{\prime}+2}\right)=(1,0),
\end{array}\right.
$$

and if $\nu^{\prime}+2<\nu$, then and so on. Thus by induction on $j$ we see that for $\nu^{\prime}<j \leq \nu$ we have

$$
\left(x_{j}, y_{j}\right)=\left(x_{j-1} / y_{j-1}, y_{j-1}\right) \text { with }\left(\operatorname{ord}_{U^{\prime}} x_{j}, \operatorname{ord}_{U^{\prime}} y_{j}\right)=(1,0) .
$$

Hence, letting $T^{\prime}$ be the unique member of $Q_{1}\left(S_{\nu}\right)$ with $M\left(T^{\prime}\right)=\left(x_{\nu} / y_{\nu}, y_{\nu}\right) T^{\prime}$, we see that $T^{\prime} \subset U^{\prime}$ and for all $T^{*} \in Q_{1}\left(S_{\nu}\right) \backslash\left\{T^{\prime}\right\}$ we have $T^{*} \notin U^{\prime}$.

Proof of (4.4.5). Let $U^{\prime}=o\left(S_{\nu^{\prime}-1}\right)$. Now

$$
\left\{\begin{array}{l}
U^{\prime}=o\left(S_{\nu^{\prime}-1}\right) \text { with } y_{\nu^{\prime}}=y_{\nu^{\prime}-1} \\
\Rightarrow\left(\operatorname{ord}_{U^{\prime}} x_{\nu^{\prime}}, \operatorname{ord}_{U^{\prime}} y_{\nu^{\prime}}\right)=(0,1)
\end{array}\right.
$$


and

$$
\left\{\begin{array}{l}
\left(\operatorname{ord}_{U^{\prime}} x_{\nu^{\prime}}, \operatorname{ord}_{U^{\prime}} y_{\nu^{\prime}}\right)=(0,1) \text { and } S_{\nu^{\prime}} \subset U^{\prime} \text { with } y_{\nu^{\prime}+1}=y_{\nu^{\prime}} / x_{\nu^{\prime}} \\
\Rightarrow\left(\operatorname{ord}_{U^{\prime}} x_{\nu^{\prime}+1}, \operatorname{ord}_{U^{\prime}} y_{\nu^{\prime}+1}\right)=(0,1) \text { and } S_{\nu^{\prime}+1} \subset U^{\prime}
\end{array}\right.
$$

and

$$
\left\{\begin{array}{l}
\left(\operatorname{ord}_{U^{\prime}} x_{\nu^{\prime}+1}, \operatorname{ord}_{U^{\prime}} y_{\nu^{\prime}+1}\right)=(0,1) \text { and } S_{\nu^{\prime}+1} \subset U^{\prime} \text { with } y_{\nu^{\prime}+2}=y_{\nu^{\prime}+1} / x_{\nu^{\prime}+1} \\
\Rightarrow\left(\operatorname{ord}_{U^{\prime}} x_{\nu^{\prime}+2}, \operatorname{ord}_{U^{\prime}} y_{\nu^{\prime}+2}\right)=(0,1) \text { and } S_{\nu^{\prime}+2} \subset U^{\prime}
\end{array}\right.
$$

and so on. Thus by induction on $j$ we see that for $\nu^{\prime}<j \leq \nu$ we have

$$
\left(\operatorname{ord}_{U^{\prime}} x_{j}, \operatorname{ord}_{U^{\prime}} y_{j}\right)=(0,1) \text { and } S_{j} \subset U^{\prime} .
$$

Hence, letting $T^{\prime}$ be the unique member of $Q_{1}\left(S_{\nu}\right)$ with $M\left(T^{\prime}\right)=\left(x_{\nu}, y_{\nu} / x_{\nu}\right) T^{\prime}$, we see that $T^{\prime} \subset U^{\prime}$ and for all $T^{*} \in Q_{1}\left(S_{\nu}\right) \backslash\left\{T^{\prime}\right\}$ we have $T^{*} \notin U^{\prime}$.

Proof of (4.4.6). Let $U^{\prime}=o\left(S_{\nu^{\prime}-1}\right)$. Now

$$
\left\{\begin{array}{l}
U^{\prime}=o\left(S_{\nu^{\prime}-1}\right) \text { with } x_{\nu^{\prime}}=x_{\nu^{\prime}-1} \\
\Rightarrow\left(\operatorname{ord}_{U^{\prime}} y_{\nu^{\prime}}, \operatorname{ord}_{U^{\prime}} x_{\nu^{\prime}}\right)=(0,1)
\end{array}\right.
$$

and

$$
\left\{\begin{array}{l}
\left(\operatorname{ord}_{U^{\prime}} y_{\nu^{\prime}}, \operatorname{ord}_{U^{\prime}} x_{\nu^{\prime}}\right)=(0,1) \text { and } S_{\nu^{\prime}} \subset U^{\prime} \text { with } x_{\nu^{\prime}+1}=x_{\nu^{\prime}} / y_{\nu^{\prime}} \\
\Rightarrow\left(\operatorname{ord}_{U^{\prime}} y_{\nu^{\prime}+1}, \operatorname{ord}_{U^{\prime}} x_{\nu^{\prime}+1}\right)=(0,1) \text { and } S_{\nu^{\prime}+1} \subset U^{\prime}
\end{array}\right.
$$

and

$$
\left\{\begin{array}{l}
\left(\operatorname{ord}_{U^{\prime}} y_{\nu^{\prime}+1}, \operatorname{ord}_{U^{\prime}} x_{\nu^{\prime}+1}\right)=(0,1) \text { and } S_{\nu^{\prime}+1} \subset U^{\prime} \text { with } x_{\nu^{\prime}+2}=x_{\nu^{\prime}+1} / y_{\nu^{\prime}+1} \\
\Rightarrow\left(\operatorname{ord}_{U^{\prime}} y_{\nu^{\prime}+2}, \operatorname{ord}_{U^{\prime}} x_{\nu^{\prime}+2}\right)=(0,1) \text { and } S_{\nu^{\prime}+2} \subset U^{\prime}
\end{array}\right.
$$

and so on. Thus by induction on $j$ we see that for $\nu^{\prime}<j \leq \nu$ we have

$$
\left(\operatorname{ord}_{U^{\prime}} y_{j}, \operatorname{ord}_{U^{\prime}} x_{j}\right)=(0,1) \text { and } S_{j} \subset U^{\prime} .
$$

Hence, letting $T^{\prime}$ be the unique member of $Q_{1}\left(S_{\nu}\right)$ with $M\left(T^{\prime}\right)=\left(y_{\nu}, x_{\nu} / y_{\nu}\right) T^{\prime}$, we see that $T^{\prime} \subset U^{\prime}$ and for all $T^{*} \in Q_{1}\left(S_{\nu}\right) \backslash\left\{T^{\prime}\right\}$ we have $T^{*} \notin U^{\prime}$.

Lemma 4.5. Upon letting $V=o\left(S_{\nu}\right)$ and

$$
J=\left\{j \in \mathbb{N}: j<\nu \text { and } T \subset o\left(S_{j}\right) \text { for some } T \in \pi(R, V)\right\},
$$

we have the following.

(4.5.1). Assume that $\nu=0$. Then $J=\emptyset$ and $\pi^{*}(R, V)=\pi(R, V)$.

(4.5.2). Assume that $t(S)=\nu>0$. Then $J=\{\nu-1\}$, and letting $T$ be the unique member of $Q_{1}\left(S_{\nu}\right)$ with $M(T)=\left(x_{\nu} / y_{\nu}, y_{\nu}\right) T$, we have $\pi(R, V) \backslash \pi^{*}(R, V)=\{T\}$ and $T \subset o\left(S_{\nu-1}\right)$.

(4.5.3). Assume that $t(S)<\nu>0$ and for every integer $j$ with $t(S)<j \leq \nu$ we have that $j$ is an $X$-operation of $S$. Then $J=\{\nu-1\}$, and letting $T$ be the unique member of $Q_{1}\left(S_{\nu}\right)$ with $M(T)=\left(x_{\nu} / y_{\nu}, y_{\nu}\right) T$, we have $\pi(R, V) \backslash \pi^{*}(R, V)=\{T\}$ and $T \subset o\left(S_{\nu-1}\right)$.

(4.5.4). Assume that $t(S)<\nu>0$ and for every integer $j$ with $t(S)<j \leq \nu$ we have that $j$ is a $Y$-operation of $S$. Then $J=\{\nu-1\}$, and letting $T$ be the unique member of $Q_{1}\left(S_{\nu}\right)$ with $M(T)=\left(x_{\nu}, y_{\nu} / x_{\nu}\right) T$, we have $\pi(R, V) \backslash \pi^{*}(R, V)=\{T\}$ and $T \subset o\left(S_{\nu-1}\right)$.

(4.5.5). Assume that $\nu>\nu^{\prime}>0$ for an integer $\nu^{\prime}$ such that $\nu^{\prime}$ is a $Y$-operation of $S$ and for every integer $j$ with $\nu^{\prime}<j \leq \nu$ we have that $j$ is an $X$-operation of $S$. 
Then $J=\left\{\nu^{\prime}-1, \nu-1\right\}$, and letting $T^{\prime} \neq T$ be the unique members of $Q_{1}\left(S_{\nu}\right)$ with $M\left(T^{\prime}\right)=\left(x_{\nu}, y_{\nu} / x_{\nu}\right) T^{\prime}$ and $M(T)=\left(x_{\nu} / y_{\nu}, y_{\nu}\right) T$, we have $\pi(R, V) \backslash \pi^{*}(R, V)=$ $\left\{T^{\prime}, T\right\}$ and $T^{\prime} \subset o\left(S_{\nu^{\prime}-1}\right)$ with $T^{\prime} \not \subset o\left(S_{\nu-1}\right)$ and $T \subset o\left(S_{\nu-1}\right)$ with $T \not \subset o\left(S_{\nu^{\prime}-1}\right)$.

(4.5.6). Assume that $\nu>\nu^{\prime}>0$ for an integer $\nu^{\prime}$ such that $\nu^{\prime}$ is an $X$-operation of $S$ and for every integer $j$ with $\nu^{\prime}<j \leq \nu$ we have that $j$ is a $Y$-operation of $S$. Then $J=\left\{\nu^{\prime}-1, \nu-1\right\}$, and letting $T^{\prime} \neq T$ be the unique members of $Q_{1}\left(S_{\nu}\right)$ with $M\left(T^{\prime}\right)=\left(x_{\nu} / y_{\nu}, y_{\nu}\right) T^{\prime}$ and $M(T)=\left(x_{\nu}, y_{\nu} / x_{\nu}\right) T$, we have $\pi(R, V) \backslash \pi^{*}(R, V)=$ $\left\{T^{\prime}, T\right\}$ and $T^{\prime} \subset o\left(S_{\nu^{\prime}-1}\right)$ with $T^{\prime} \not \subset o\left(S_{\nu-1}\right)$ and $T \subset o\left(S_{\nu-1}\right)$ with $T \not \subset o\left(S_{\nu^{\prime}-1}\right)$.

(4.5.7). The cases (4.5.1) to (4.5.6) are mutually exclusive and exhaustive, and hence in particular $0 \leq|J|=\left|\pi(R, V) \backslash \pi^{*}(R, V)\right| \leq 2$ and for every $j \in J$ there is a unique $T_{j} \in \pi(R, V) \backslash \pi^{*}(R, V)$ with $T_{j} \subset o\left(S_{j}\right)$.

Proof. Follows from (4.4).

Lemma 4.6. Concerning the multiplicity and glue sequences associated with $S$ we have the following.

(4.6.1). If $t(S)>0$, then

$$
m_{0}(S) \geq m_{1}(S) \geq \cdots \geq m_{t(S)-1}(S)=1,
$$

and for $0 \leq j \leq t(S)-1$ we have

$$
m_{j}(S)=\min \left(a_{j}(S), b_{j}(S)\right) .
$$

(4.6.2). We have

$$
m_{0}^{* *}(S) \geq m_{1}^{* *}(S) \geq \cdots \geq m_{t(S)-1}^{* *}(S)=1,
$$

and for $0 \leq j \leq \nu$ we have

$$
m_{j}^{* *}(S)=\min \left(a_{j}^{* *}(S), b_{j}^{* *}(S)\right) .
$$

(4.6.3). We have

$$
m_{0}^{*}(S, 1,1) \geq m_{1}^{*}(S, 1,1) \geq \cdots \geq m_{\nu}^{*}(S, 1,1)=1,
$$

and for $0 \leq j \leq \nu$ we have

$$
m_{j}^{*}(S, 1,1)=\min \left(a_{j}^{*}(S, 1,1), b_{j}^{*}(S, 1,1)\right) .
$$

Proof of (4.6.1). The special cases when $S$ is either a flip or a nonflip with $\nu>0$ can be checked directly, and then the general case follows from the special cases.

Proof of (4.6.2). Follows from (4.6.1).

Proof of (4.6.3). Follows from (4.6.1).

Theorem 4.7. Let $\left(F_{j}, I_{j}, d_{j}\right)_{0 \leq j \leq \nu}$ be the sprout on $S$ described at the end of Section 2. Let $\mu=\nu+1$. Let $\left(S_{\mu}, x_{\mu}, y_{\nu}, \kappa_{\mu}\right)$ be a QDT of $\left(S_{\nu}, x_{\nu}, y_{\nu}, \kappa_{\nu}\right)$ such that upon letting $I_{\mu}=F_{\mu} S_{\mu}$ and $d_{\mu}=\operatorname{ord}_{S_{\mu}} I_{\mu}$ where $0 \neq F_{\mu} \in S_{\mu}$ is such that

$$
F_{\nu} / F_{\mu}=z_{\nu}^{d_{\nu}} \text { with } z_{\nu}= \begin{cases}x_{\nu} & \text { if } x_{\mu}=x_{\nu} \\ y_{\nu} & \text { if } y_{\mu}=y_{\nu},\end{cases}
$$

we have that $I_{\mu} \neq S_{\mu}$ and $\left(S_{\nu}, S_{\mu}^{\prime}\right)\left(I_{\nu}\right)=S_{\mu}^{\prime}$ whenever $S_{\mu}^{\prime} \in Q_{1}\left(S_{\nu}\right) \backslash\left\{S_{\mu}\right\}$. Note that now $S^{\dagger}=\left(S_{j}, x_{j}, y_{j}, \kappa_{j}\right)_{0 \leq j \leq \mu}$ is a finite QDT sequence of $(R, x, y, \kappa)$ and $\left(F_{j}, I_{j}, d_{j}\right)_{0 \leq j \leq \mu}$ is a sprout on $S^{\dagger}$. For $0 \leq j \leq \mu$ let $N\left(F_{j}\right)$ be the Newton polygon 
of $F_{j}$ relative to $\left(S_{j}, x_{j}, y_{j}, \kappa_{j}\right)$. Let $V=o\left(S_{\nu}\right)$. Assume that $I_{\mu} \in \gamma\left(R, S_{\mu}, V\right)$ and let $\theta=\theta\left(R, S_{\mu}, I_{\mu}\right)$. For $0 \leq j \leq \nu$ let

$$
\left(m_{j}^{*}, a_{j}^{*}, b_{j}^{*}\right)=\left(m_{j}^{*}(S, 1,1), a_{j}^{*}(S, 1,1), b_{j}^{*}(S, 1,1)\right) .
$$

Then we have the following.

(4.7.1). Assume that $\mu$ is a translation of $S^{\dagger}$. Then for $0 \leq j \leq \nu$ we have that $d_{j}=m_{j}^{*} \theta$ and $N\left(F_{j}\right)$ is a triangle with top point $\left(0, a_{j}^{*} \theta\right)$ and bottom point $\left(b_{j}^{*} \theta, 0\right)$.

(4.7.2). Assume that $\mu$ is an $X$-operation of $S^{\dagger}$ and $S_{\mu} \in \pi^{*}(R, V)$. Then $\chi\left(S_{\nu}, S_{\mu}\right)=1, d_{\mu}=\theta, t\left(S^{\dagger}\right)=t(S) \leq \nu$, and for every integer $j$ with $t(S)<j \leq \nu$ we have that $j$ is an $X$-operation of $S$. Moreover: (1) for $0 \leq j \leq \nu$ we have $d_{j}=m_{j}^{*} \theta$, (2) for $t(S) \leq j \leq \nu$ we have that the first point of $N\left(F_{j}\right)$ is $(0, \theta)$, and (3) for $0 \leq j \leq t(S)-1$ we have that $N\left(F_{j}\right)$ is a triangle with top point $\left(0, a_{j}^{*} \theta\right)$ and bottom point $\left(b_{j}^{*} \theta, 0\right)$. Furthermore, if $I_{\mu}=y_{\mu} S_{\mu}$, then: (2*) for $t(S) \leq j \leq \nu$ we have $I_{j}=y_{j} S_{j}$.

(4.7.3). Finally assume that $\mu$ is a $Y$-operation of $S^{\dagger}$ and $S_{\mu} \in \pi^{*}(R, V)$. Then $\chi\left(S_{\nu}, S_{\mu}\right)=1, d_{\mu}=\theta, t\left(S^{\dagger}\right)=t(S) \leq \nu$, and for every integer $j$ with $t(S)<j \leq \nu$ we have that $j$ is a $Y$-operation of $S$. Moreover: (1) for $0 \leq j \leq \nu$ we have $d_{j}=m_{j}^{*} \theta$, (2) for $t(S) \leq j \leq \nu$ we have that the last point of $N\left(F_{j}\right)$ is $(\theta, 0)$, and (3) for $0 \leq j \leq t(S)-1$ we have that $N\left(F_{j}\right)$ is a triangle with top point $\left(0, a_{j}^{*} \theta\right)$ and bottom point $\left(b_{j}^{*} \theta, 0\right)$. Furthermore, if $I_{\mu}=x_{\mu} S_{\mu}$ then: (2*) for $t(S) \leq j \leq \nu$ we have $I_{j}=x_{j} S_{j}$.

Proof of (4.7.1). In view of (3.1.5), by $(2 \mathrm{~A})$ to $(2 \mathrm{C})$ of $\mathrm{Ab13}$ ] we see that $N\left(F_{\nu}\right)$ is a triangle with top point $(0, \theta)$ and bottom point $(\theta, 0)$. Therefore, by $(4.2 .1)$, for $0 \leq j \leq \nu$ we have that $d_{j}=m_{j}^{*} \theta$ and $N\left(F_{j}\right)$ is a triangle with top point $\left(0, a_{j}^{*} \theta\right)$ and bottom point $\left(b_{j}^{*} \theta, 0\right)$.

Proof of (4.7.2). In view of (3.1.1) to (3.1.5), by (2A) to (2C) of Ab13 we see that $\chi\left(S_{\nu}, S_{\mu}\right)=1, d_{\mu}=\theta, t\left(S^{\dagger}\right)=t(S) \leq \nu$, and the first point of $N\left(F_{\nu}\right)$ is $(0, \theta)$. Now by (4.4) we conclude that for every integer $j$ with $t(S)<j \leq \nu$ we have that $j$ is an $X$-operation of $S$. Therefore by (4.2) we get the rest.

Proof of (4.7.3). In view of (3.1.1) to (3.1.5), by (2A) to (2C) of [Ab13] we see that $\chi\left(S_{\nu}, S_{\mu}\right)=1, d_{\mu}=\theta, t\left(S^{\dagger}\right)=t(S) \leq \nu$, and the last point of $N\left(F_{\nu}\right)$ is $(\theta, 0)$. Now by (4.4) we conclude that for every integer $j$ with $t(S)<j \leq \nu$ we have that $j$ is a $Y$-operation of $S$. Therefore by (4.2) we get the rest.

Definition 4.8. We put:

$\sigma(R)=$ set of all stems of $R$, i.e., all finite sequences $\bar{S}=\left(\bar{S}_{j}, I_{j}\right)_{0 \leq j \leq \mu}$ where $\left(\bar{S}_{j}\right)_{0 \leq j \leq \mu}$ is a finite QDT sequence of $R$ with $I_{j} \in P^{\times}\left(\bar{S}_{j}\right)$ for $0 \leq j \leq \mu$ such that for $0 \leq j<\mu$ we have $\left(\bar{S}_{j}, \bar{S}_{j+1}\right)\left(I_{j}\right)=I_{j+1}$, and $\left(\bar{S}_{j}, \bar{S}_{j+1}^{\prime}\right)\left(I_{j}\right)=\bar{S}_{j+1}^{\prime}$ whenever $\bar{S}_{j+1}^{\prime} \in Q_{1}\left(\bar{S}_{j}\right) \backslash\left\{\bar{S}_{j+1}\right\}$.

For any $V \in D(R)^{\Delta}$ we put:

$\sigma(R, V)=$ set of all arc stems of $(R, V)$, i.e., all members $\bar{S}=\left(\bar{S}_{j}, I_{j}\right)_{0 \leq j \leq \mu}$ of $\sigma(R)$ such that $\left(\bar{S}_{j}\right)_{0 \leq j \leq \mu-1}$ is the finite QDT sequence of $R$ along $V$ with $I_{\mu} \in \gamma\left(R, \bar{S}_{\mu}, V\right)$. We define the weight of $\bar{S}$ by letting

$$
\theta(R, \bar{S})=\theta\left(R, \bar{S}_{\mu}, I_{\mu}\right)
$$


and we put

$$
\sigma^{*}(R, V)=\left\{\begin{array}{l}
\left\{\bar{S}=\left(\bar{S}_{j}, I_{j}\right)_{0 \leq j \leq \mu} \text { in } \sigma(R, V): I_{\mu} \in \gamma^{*}\left(R, \bar{S}_{\mu}, V\right)\right\} \\
=\text { set of all curvette stems of }(R, V)
\end{array}\right.
$$

where we call $\mu$ the layer index of $V$ over $R$ and denote it by $\mu(R, V)$, and we note that $V \in D_{\mu-1}(R)^{\Delta}$.

Theorem 4.9. For any $V \in D(R)^{\Delta}$ let $\bar{S}=\left(\bar{S}_{j}, I_{j}\right)_{0 \leq j \leq \mu}$ be any member of $\sigma^{*}(R, V)$. Assume that $\bar{S}_{j}=S_{j}$ for $0 \leq j \leq \nu$ with $\nu=\mu-1$. Then for $0 \leq j \leq \nu$ we have $\operatorname{ord}_{S_{j}} I_{j}=m_{j}^{*}(S, 1,1) \theta(R, \bar{S})$, where $m^{*}(S, 1,1)=m_{j}^{*}(S, 1,1)_{0 \leq j \leq \nu}$ is a sequence of positive integers with

$$
m_{0}^{*}(S, 1,1) \geq m_{1}^{*}(S, 1,1) \geq \cdots \geq m_{\nu}^{*}(S, 1,1)=1 .
$$

Proof. Follows from (4.6.3) and (4.7).

\section{Redoing the multiplicity SEQuence}

Again let $x, y$ be generators of the maximal ideal $M(R)$ of a two dimensional regular local domain $R, \kappa$ be a coefficient set of $R$, and let $S=\left(S_{j}, x_{j}, y_{j}, \kappa_{j}\right)_{0 \leq j \leq \nu}$ be a finite QDT sequence of $(R, x, y, \kappa)$. Leaving the checking to the reader, here is a concise derivation of the positive integers $\left(m_{j}(S), a_{j}(S), b_{j}(S)\right)_{0 \leq j \leq t(S)-1}$ where we are assuming $t(S)>0$. The initial condition $\left(m_{t(S)-1}, a_{t(S)-1}, b_{t(S)-1}\right)=(1,1,1)$ is followed by the recurrence formula

$$
\left(m_{j-1}, a_{j-1}, b_{j-1}\right)= \begin{cases}\left(a_{j}, a_{j}, a_{j}+b_{j}\right) & \text { if } j \text { is an } X \text {-operation } \\ \left(b_{j}, a_{j}+b_{j}, b_{j}\right) & \text { if } j \text { is a } Y \text {-operation } \\ \left(a_{j} \chi_{j}, a_{j} \chi_{j}, a_{j} \chi_{j}\right) & \text { if } j \text { is a translation }\end{cases}
$$

where $\chi_{j}=\chi\left(S_{j-1}, S_{j}\right)$ for $t(S)<j \leq \nu$ with decreasing induction on $j$.

\section{REFERENCES}

[Ab1] Shreeram Abhyankar, On the valuations centered in a local domain, Amer. J. Math. 78 (1956), 321-348. MR0082477 (18,556b)

[Ab2] Shreeram Abhyankar, Ramification theoretic methods in algebraic geometry, Annals of Mathematics Studies, no. 43, Princeton University Press, Princeton, N.J., 1959. MR.0105416 (21 \#4158)

[Ab3] S. S. Abhyankar, Resolution of singularities of embedded algebraic surfaces, 2nd ed., Springer Monographs in Mathematics, Springer-Verlag, Berlin, 1998. MR.1617523 (99c:14021)

[Ab4] Shreeram S. Abhyankar, Historical ramblings in algebraic geometry and related algebra, Amer. Math. Monthly 83 (1976), no. 6, 409-448. MR0401754 (53 \#5581)

[Ab5] Shreeram S. Abhyankar, Desingularization of plane curves, Singularities, Part 1 (Arcata, Calif., 1981), Proc. Sympos. Pure Math., vol. 40, Amer. Math. Soc., Providence, RI, 1983, pp. 1-45. MR713043 (85d:14024)

[Ab6] Shreeram S. Abhyankar, Algebraic geometry for scientists and engineers, Mathematical Surveys and Monographs, vol. 35, American Mathematical Society, Providence, RI, 1990. MR.1075991(92a:14001)

[Ab7] Shreeram S. Abhyankar, Polynomial expansion, Proc. Amer. Math. Soc. 126 (1998), no. 6, 1583-1596, DOI 10.1090/S0002-9939-98-04183-5. MR.1443142 (98g:12003)

[Ab8] S. S. Abhyankar, Lectures on Algebra. I, World Scientific, 2006. MR2253320 (2007k:13001)

[Ab9] Shreeram S. Abhyankar, Inversion and invariance of characteristic terms: Part I, The legacy of Alladi Ramakrishnan in the mathematical sciences, Springer, New York, 2010, pp. 93-168, DOI 10.1007/978-1-4419-6263-8_8. MR2744259(2012b:14057) 
[Ab10] Shreeram S. Abhyankar, Dicritical divisors and Jacobian problem, Indian J. Pure Appl. Math. 41 (2010), no. 1, 77-97, DOI 10.1007/s13226-010-0017-x. MR2650101 (2011d:14107)

[Ab11] Shreeram S. Abhyankar, Pillars and towers of quadratic transformations, Proc. Amer. Math. Soc. 139 (2011), no. 9, 3067-3082, DOI 10.1090/S0002-9939-2011-10731-7. MR2811263 (2012f:14003)

[Ab12] Shreeram S. Abhyankar, More about dicriticals, Proc. Amer. Math. Soc. 139 (2011), no. 9, 3083-3097, DOI 10.1090/S0002-9939-2011-10732-9. MR2811264(2012d:13047)

[Ab13] Shreeram S. Abhyankar, Quadratic transforms inside their generic incarnations, Proc. Amer. Math. Soc. 140 (2012), no. 12, 4111-4126, DOI 10.1090/S0002-9939-2012-11583-7. MR.2957201

[Ab14] S. S. Abhyankar, Dicritical divisors and Dedekind's Gauss lemma, Proc. Amer. Math. Soc., to appear.

[AA1] S. S. Abhyankar and E. Artal Bartolo, Curvettes and dicritical divisors, Proc. Amer. Math. Soc., to appear.

[AH1] Shreeram S. Abhyankar and William J. Heinzer, Existence of dicritical divisors, Amer. J. Math. 134 (2012), no. 1, 171-192, DOI 10.1353/ajm.2012.0002. MR2876143

[AH2] Shreeram S. Abhyankar and William J. Heinzer, Existence of dicritical divisors revisited, Proc. Indian Acad. Sci. Math. Sci. 121 (2011), no. 3, 267-290, DOI 10.1007/s12044-0110035-6. MR2867982 (2012h:14148)

[AL1] Shreeram S. Abhyankar and Ignacio Luengo, Algebraic theory of dicritical divisors, Amer. J. Math. 133 (2011), no. 6, 1713-1732, DOI 10.1353/ajm.2011.0045. MR2863374

[Art] E. Artal-Bartolo, Une démonstration géométrique du théorème d'Abhyankar-Moh, J. Reine Angew. Math. 464 (1995), 97-108, DOI 10.1515/crll.1995.464.97 (French). MR $1340336(96 \mathrm{k}: 14008)$

Department of Mathematics, Purdue University, West Lafayette, Indiana 47907

E-mail address: ram@cs.purdue.edu

Facultad de Matematicas, Universidad Complutense de Madrid, 28040 Madrid, Spain

E-mail address: iluengo@mat.ucm.es 